\title{
Effects of Oxalic Acid on Physiological-biochemical Traits of Cold Temperate Larix gmelinii Seedlings under Soil Nutrient Deficiency
}

\author{
Fu-Xing CUI ${ }^{1,2}$, Jin-Feng SONG ${ }^{1}$, Dao-Guang $\mathrm{ZHU}^{2}$, Jin-Bo $\mathrm{LI}^{1,2}$, \\ Zhen-Yang $\mathrm{QI}^{3}$, Lin SHAN ${ }^{1}$, Hong-Wei $\mathrm{NI}^{2, *}$ \\ ${ }^{1}$ Northeast Forestry University, Heilongjiang Harbin 150040 \\ ${ }^{2}$ Institute of natural resources and ecology of HAS, Heilongjiang Harbin 150040 \\ ${ }^{3}$ Huzhong meteorological agency, Heilongjiang Huzhong 165036) \\ ${ }^{*}$ Corresponding author
}

Keywords: Soil nutrient deficiency, Brown coniferous forest soils, Oxalic acid, Larix gmelinii seedlings, Physiological-biochemical traits.

\begin{abstract}
Organic acids obviously affect plant survival and growth under soil nutrient deficiency condition. Variable proportion $\mathrm{A}_{1}$ and $\mathrm{C}$ horizon mixed cold temperate brown coniferous forest soils was applied to make soil nutrient deficiency condition, and then Larix gmelinii seedlings were planted in these soils. On the basis of oxalic acid concentration range of forest litter leacheate at the northeast forest region, oxalic acid solution with different concentration was sprayed on the surface of Larix gmelinii seedlings, and the effects of oxalic acid on physiological-biochemical traits of cold temperate Larix gmelinii seedlings were studied in our experiment. Results showed that the electrical conductivity and MDA content of Larix gmelinii seedlings were decreased with the increase of oxalic acid concentration. Most SOD and POD activity of Larix gmelinii seedlings were increased. $5.0 \mathrm{mmol} \cdot \mathrm{L}^{-1}$ oxalic acid has significant effects on proline and soluble protein osmotic adjustment substances. And Larix gmelinii seedlings grew well at the concentration of $10.0 \mathrm{mmol} \cdot \mathrm{L}^{-1}$ oxalic acid.
\end{abstract}

\section{Introduction}

Soil nutrient as an impact factor plays an important role in soil fertility. Soil nutrient deficiency restricts plant growth and biomass accumulation directly, so far as to plants damage and survival. So it is a multidisciplinary hotspot about plant response and adaptation regularity to soil nutrient deficiency all the time. organic acids (low molecular organic acids) is a ubiquitous substances in forest ecosystem, whose vital function that highly accelerates soil nutritive substances activation and release, and augments plants availability [1]. Larix gmelinii is a crucial native tree in northeast forest region, and due to its high surviving and adaptive rate, therefore, it is regarded as afforestation pioneer and optimizing tree species of forestry reclamation in northeast area. However, the survival and growth of Larix gmelinii is still limited at the serious soil nutrient deficiency. Hence, it is important to improve Larix gmelinii resistance to above condition.

In our study, we made nutrient deficiency soil and transplanted Larix gmelinii seedlings, and sprayed simulated appropriate oxalic acid on seedlings repeatedly. And effects of oxalic acid on physiological-biochemical traits of cold temperate Larix gmelinii seedlings under soil nutrient deficiency was studied in order to find feasibility 
mechanism that oxalic acid improve the resistance of Larix gmelinii seedlings to soil nutrient deficiency, and improve infertile soil and the quality of forest establishment.

\section{Materials and Methods}

Experiment was conducted in Huzhong National Natural Reserve of Great Khingan Mountains. Firstly, $\mathrm{A}_{1}$ and $\mathrm{C}$ horizon cold temperate brown coniferous forest soil(the proportion of $\mathrm{A}_{1}$ and $\mathrm{C}$ horizon soil equals $50 \%$ respectively) was mixed to make soil nutrient deficiency condition, and then Larix gmelinii seedlings were transplanted into soil nutrient deficiency soil. During seedlings recovering with normal illumination and water in greenhouse, the average height and ground diameter of seedlings were $4.3 \mathrm{~cm}$ and $1.05 \mathrm{~cm}$, respectively. Secondly, we prepared oxalic acid solution refer to local representative forest litter leacheate concentration [2], including 0, 0.2, 1.0, 5.0, 10.0 $\mathrm{mmol} / \mathrm{L}$, and $0 \mathrm{mmol} / \mathrm{L}$ oxalic acid as control. And seedlings were sprayed uniformly with above different oxalic acids at 18:00 until surface was moist, one time per 4 days, totally 7 times. In the end, 11 20 mature needles were collected randomly after 30 days, and was determined their relative electrolytic leakage, malonaldehyde (MDA), superoxide dismutase (SOD), peroxidase (POD), proline (Pro), soluble protein instantly. And the relative electrolytic leakage was measured by Shanghai DDS-6700 conductometer, MDA content was measured with thiobarbituric acid chromatometry (TBA) method, SOD and POD activity were measured by nitroblue tetrazolium photochemical reduction (NBT) method and guaiacol colorimetric method respectively, and Pro content and soluble protein content were measured by acidic ninhydrin method and Coomassie brilliant blue G-250 staining method. Each process repeats 3 times.

\section{Results}

\section{Effect of Oxalic Acid on Larix gmelinii Seedlings Cellular Membrane System under Soil Nutrient Deficiency Condition}

After oxalic acid treatment, the relative electrolytic leakage of Larix gmelinii seedlings was gradually decreased with the rise of oxalic acid concentration in the range of $0.2 \sim 10.0 \mathrm{mmol} \cdot \mathrm{L}^{-1}$, it suggested the cell membrane permeability was gradually decreased, and this illustrated that high oxalic acid concentration treatment had opposite effect on resisting seedlings electrolyte effusion under soil nutrient deficiency (Fig 1a). MDA content of Larix gmelinii seedlings needles were also gradually decreased with the rise of oxalic acid concentration, and the decreasing amplitude reach $3.57 \% \sim 14.28 \%$ (Fig 1b), and there was a significantly variation among all the MDA value with different concentration treatments $(\mathrm{P}>0.1)$ (Table 1$)$. It showed the more oxalic acid was, the more peroxidation damage of cellular membrane system was under soil nutrient deficiency. 




Figure 1(1) The relative electrolytic leakage change

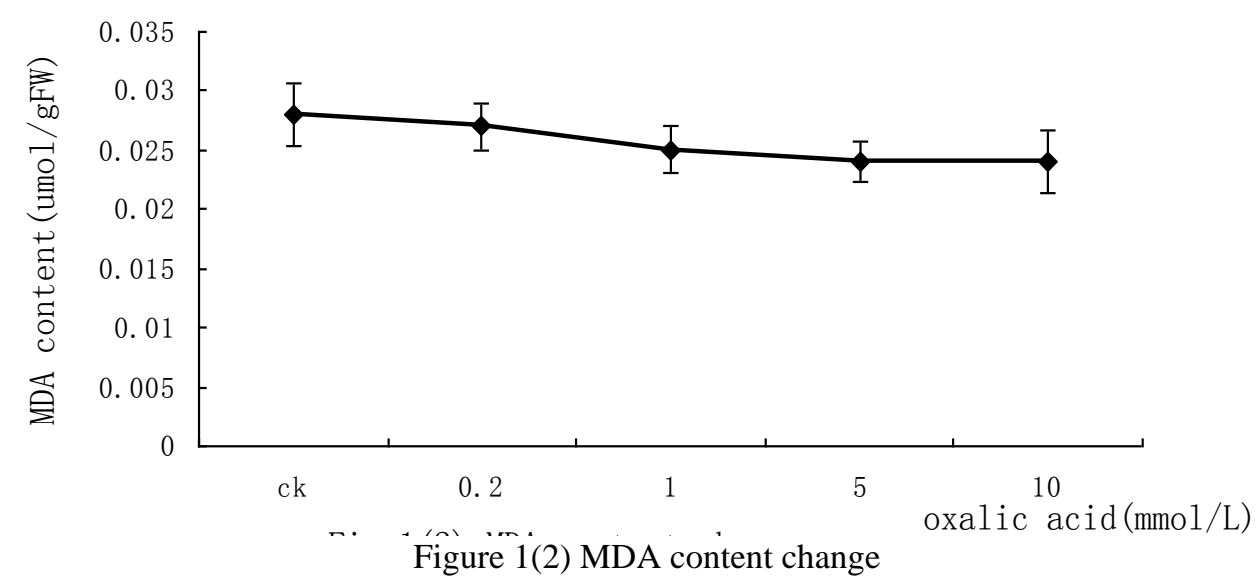

Figure 1. Oxalic acid effect on the relative electrolytic leakage of Larix gmelinii seedlings needle and MDA content under soil nutrient deficiency

\section{Effect of Oxalic Acid on Larix gmelini Seedlings Antioxidant Enzyme Activity under Soil Nutrient Deficiency Condition}

Experimental results showed that both the SOD and POD value of Larix gmelinii seedlings gradually went up after oxalic acid treatments, it indicated that SOD and POD activity of seedlings were gradually enhanced by their own enzymatic defense mechanism, which could alleviate membrane lipid peroxide level and resist the damage of soil nutrient deficiency. Oxalic acid has obvious effect on the seedlings SOD activity under soil nutrient deficiency. When the oxalic acid concentration was $1 \mathrm{mmol} \cdot \mathrm{L}^{-1}$, SOD activity was most obvious, and the increasing amplitude was $9.4 \%$. It generally presented a peak curve changes between SOD activity and oxalic acid concentration, which rose first and fell later, and the value of seedlings SOD activity was higher than that without oxalic acid treatment all the way. On the other hand, the seedlings POD activity were enhanced by the addition of oxalic acid treatments under soil nutrient deficiency, it was most obvious for seedlings POD activity when the concentration of oxalic acid was at $10 \mathrm{mmol} \cdot \mathrm{L}^{-1}$, the increasing amplitude even was $70.7 \%$, and POD activity generally appeared the rise trend with the increase of oxalic acid concentration (Fig2). 


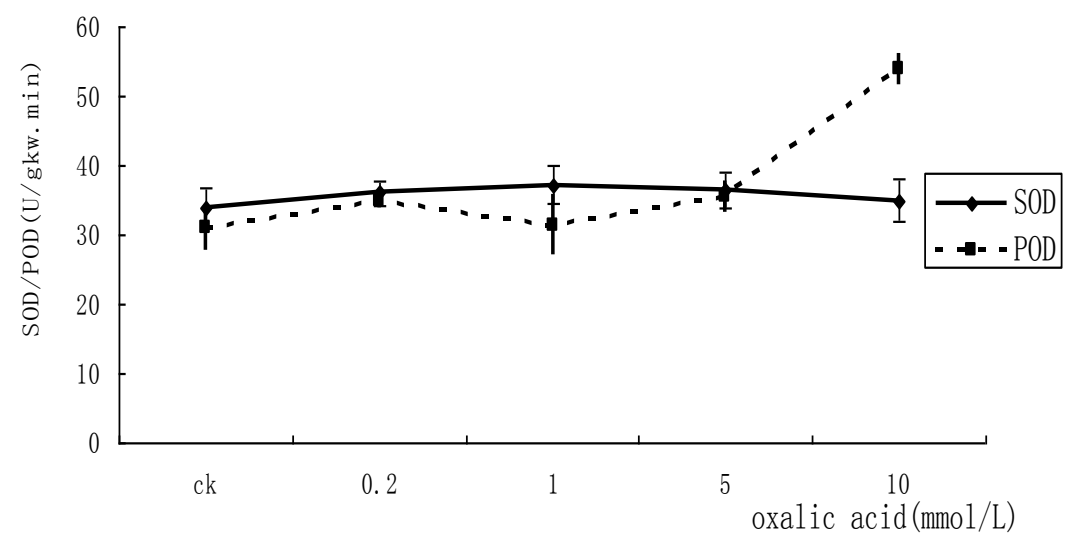

Figure 2. Oxalic acid effect on Larix gmelini seedlings antioxidant enzyme activity under soil nutrient deficiency condition

\section{Effect of Oxalic Acid on Larix gmelini Seedlings Osmotica Content under Soil Nutrient Dificiency Condition}

Proline content change without obvious rule after oxalic acid treatment, and the increase efficiency was obvious at $5.0 \mathrm{mmol} \cdot \mathrm{L}^{-1}$ concentration. It demonstrated that oxalic acids with proper concentration played an active role in Larix gmelini proline accumulation under soil nutrient deficiency, and there was an obvious effect that oxalic acids improve seedlings adaptation under nutrient dificiency. The change of Larix gmelini soluble protein content was similar with that of proline by adding oxalic acids, the value of soluble protein content also reached the peak at the oxalic acids concentration of $5.0 \mathrm{mmol} \cdot \mathrm{L}^{-1}$, and the value was decreasing after oxalic acid $5.0 \mathrm{mmol} \cdot \mathrm{L}^{-1}$ treatment. To sum up, the most obvious effect of oxalic acid on Larix gmelini seedlings osmotica was at the concentration of $5.0 \mathrm{mmol} \cdot \mathrm{L}^{-1}$ (Fig.3).



Figure 3. Oxalic acid effect of on Larix gmelini seedlings osmotica content under soil nutrient dificiency

\section{Effect of Oxalic Acid on Larix Gmelini Seedlings Growth and Biomass Accumulation under Nutrient Dificiency Condition}

Oxalic acid has some effect on the growth of Larix gmelini seedlings under nutrient dificiency condition. In our study, the growth rate of seedlings height and basal diameter increased more quickly than CK group when the oxalic acids concentration was at $0.2 \sim 10.0 \mathrm{mmol} / \mathrm{L}$, it illustrated that oxalic acids played an active role in the growth of Larix gmelini seedlings. From the perspective of oxalic acids concentration, the growth rate increased with the rise of oxalic acids concentration, and Larix gmelini 
seedlings growth and biomass accumulation was most remarkable when the oxalic acids concentration was at $10.0 \mathrm{mmol} \cdot \mathrm{L}^{-1}$ (Fig.4).



Fig4. Oxalic acid effect on growth rate of basal diameter under nutrient dificiency condition

\section{Discussion}

Oxalic acid affected the cytomembrane system of Larix gmelini seedlings was studied under soil nutrient dificiency condition in our experiment, including conductivity and MDA content, which were regarded as important indicators of plant resistance and lipid peroxidation level [3]. Under soil nutrient dificiency, plant manifested their cell membrane permeability and electrolyte leakage percentage were increased firstly, and the conductivity and MDA content dropped accordingly. It suggested high oxalic acids concentration had an opposite effect on restraining seedlings electrolyte permeation, and exacerbated cytomembrane peroxidation damage after oxalic acid treatment. Our experiment results were similar with Guo Bin [4], Drazic [5] results that salicylic acid (SA) affected plant conductivity and MDA content.

Organic acids with appropriate concentration promote various enzyme activities. Our results showed the SOD and POD activity of Larix gmelini seedlings were enhanced slightly after oxalic acid treatments, the SOD activity rose first and fell later, it generally presented a peak curve changes. all the seedlings POD activity were enhanced by the addition of oxalic acid treatments, and POD activity generally appeared the rise trend with the increase of oxalic acid concentration under soil nutrient deficiency. This results was general accordance with Yang Yan [6].

Organic acids affected plants osmotic adjustment substances, including Pro and soluble protein, etc., whose accumulation in plants reflected plants adaptive level under environmental stress. Under nutrient dificiency, we found $5.0 \mathrm{mmol} \cdot \mathrm{L}^{-1}$ oxalic acid effect was most obvious on Larix gmelini proline and soluble protein. However, Feng Xiaodong studied $1 \mathrm{mmol} / \mathrm{L}$ SA effect on jujube soluble sugar and soluble protein content was most significant [7], it demonstrated that organic acids affected plants osmotic adjustment substances due to their different types and concentration.

Seedling height and basal diameter as important morphological index could estimate plants growth. Previous studies has testified organic acids had obvious facilitation on solanum tuberosum, Brassica napus L., Cucumis sativus L, Brassica oleracea, fraxinus mandshurica, nicotiana tabacum height and leaf area [8,9]. Our experiment results showed all $0.2 \sim 10.0 \mathrm{mmol} / \mathrm{L}$ oxalic acids facilitated the growth of seedling 
height and basal diameter, the growth rate rose with the increase of concentration, and it was most significant at $10.0 \mathrm{mmol} \cdot \mathrm{L}^{-1}$ under soil nutrient dificiency.

\section{Acknowledgements}

This work was funded by Natural Science Foundation of China (31570486) Heilongjiang Province Proposed Foundation (STJB201501), Heilongjiang Province Academician Committee Foundation (201501), Youth Innovation Foundation of HAS (13FA07 14FA03 14FA10).

\section{References}

[1] Ohwaki Y, Sugauara K. Active extrusion of protons and exudation of carboxylic acids in response to iron deficiency by roots of chickpea (Cicer arietinwm L.). Plant soil, 1997, 189: 49-55.

[2] Zeng F R, Chen S, Miao Y et al. Changes of organic acid exudation and rhizosphere $\mathrm{pH}$ in rice plants under chromium stress. Environmental pollution, 2008, 155(2): 284-289 In Chinese.

[3] Li Junmei, Wang Huanxiao. Ecophysiological responses and resistance to cadmium stress in three varieties of Maize. Journal of Yunnan University, 2000, 22(4): 311-317 In Chinese.

[4] Guo Bin. Mechanisms of salicylic acid-mediated alleviation of cadmium toxicity in rice. Nanjing agricultural university, Ph.D. Thesis, 2006 In Chinese.

[5] Drazic G, Mihailovic N. Modification of cadmium toxicity in soybean seedlings by salicylic acid. Plant Sci.,2005, 168:511-517.

[6] Yang Yan. Effects of organic acids on the growth of rape under cadmium stress. Anhui normal university, master's degree thesis, 2007: 17-23 In Chinese.

[7] Feng Xiaodong, Cao Juanyun, Chen Xueli. Effect of salicylic acid on several physiological and biochemical indications in tissue culture seedling of red date. Acta Botanica Boreali-Occidentalia Sinica, 2003, 23 (9): 1625-1627.

[8] Luo Yi, Xia Guojun, Jiang Yumei. Effect of the organic acids on growth development of flue-cured tobacco. Anhui Agricultural Sciences, 2006, 34 (24): 6524-6526 In Chinese.

[9] Li keying, Li Jiaru. The Effects of Salicylic Acid on Lateral Roots Formation in Rape Seedlings. Journal of Wuhan Botanical Research, 2004, 22 (4): 345-348 In Chinese.

[10] Cui Fuxing, Song Jinfeng, Yang Di. Effects of exogenous organic acids on phosphorus availability of dark brown forest soils and phosphorus absorption and accumulation of Larix olgensis seedlings with soil nutrient deficiency. Journal of soil and water conservation, 2012, 26 (6):116-111. In Chinese.

[11] Song Jinfeng, Li Feng, Wang Yajun. Effects of exogenous organic acids on physiological and biochemical characteristics of Larix olgensis seedlings under low temperature stress. Forest Science, 2012, 48 (2):92-98. In Chinese. 